Philosophie ANTIQUE

\section{Philosophie antique}

Problèmes, Renaissances, Usages

19 | 2019

L'épicurisme antique

\title{
Frédéric FAUQUIER, Le Parménide au miroir des
}

platonismes. Logique-Ontologie-Théologie

Paris, Les Belles Lettres, 2018 (Collection d'études anciennes. Série

grecque, 157), 546 p., ISBN 978-2-251-44827-5

\section{Gwenaëlle Aubry}

\section{(2) OpenEdition}

\section{Journals}

Édition électronique

URL : https://journals.openedition.org/philosant/1790

DOI : 10.4000/philosant. 1790

ISSN : 2648-2789

Éditeur

Éditions Vrin

\section{Édition imprimée}

Date de publication : 31 octobre 2019

Pagination : 208-210

ISBN : 978-2-7574-2534-3

ISSN : $1634-4561$

\section{Référence électronique}

Gwenaëlle Aubry, «Frédéric fauauier, Le Parménide au miroir des platonismes. Logique-OntologieThéologie », Philosophie antique [En ligne], 19 | 2019, mis en ligne le 20 janvier 2019, consulté le 02 décembre 2022. URL : http://journals.openedition.org/philosant/1790 ; DOI : https://doi.org/10.4000/ philosant. 1790

\section{(c) $(1)$}

Creative Commons - Attribution - Pas d'Utilisation Commerciale - Pas de Modification 4.0 International - CC BY-NC-ND 4.0

https://creativecommons.org/licenses/by-nc-nd/4.0/ 
non à Julien, mais à sa propre présentation de l'extrait de Julien sur la limitation du pouvoir du dieu des Hébreux (CJ III, 37, 652C). Il en va de même pour le fr. 21 (p. 81) où है $\varphi \eta v$ est traduit par « d'après ce qu'il a dit ». Dans ce passage, Cyrille rappelle que son adversaire suit les doctrines de Platon en y ajoutant les siennes propres. Cyrille dit donc « comme je l'ai dit » en renvoyant à ce qu'il a soutenu quelques lignes plus haut : « il fanfaronne, s'enorgueillit et ne s'attache qu'aux seules paroles de Platon » (CJ IV, 1). Le recours aux éditions récentes aurait permis d'éviter ces erreurs. Le fr. 67 commente l'épisode mystérieux de l'union des « fils de Dieu » avec des filles des hommes (Gn 6, 2-4) invoqué pour prouver que Moïse connaît une pluralité de fils de Dieu et non un Fils unique. Or pour Julien, ces « fils de Dieu » sont des anges. Au lieu de traduire : « celui qui parle de nombreux fils de Dieu et les appelle non pas hommes mais anges », il faut comprendre : « celui qui nomme une pluralité de fils de Dieu - et il ne s'agit pas

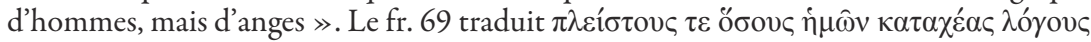
par « déverser en abondance des formules empruntées à notre doctrine », alors que le sens est « déverser contre nous la plus grande quantité possible d'arguments » (il s'agit d'une expression cyrillienne déjà utilisée en CJ I, 38 et VIII, 4).

En raison du rôle majeur de la Bible dans l'argumentation de Julien, on peut regretter l'absence de l'utilisation de G. Rinaldi, La Bibbia dei pagani, 2 vol., Bologne, 1998. De fait, comme le souligne Cyrille dans sa préface, le recours de l'empereur à la Bible donnait à son écrit une efficacité redoutable. Or l'index des lieux qui mêle toutes les références ne permet pas de se faire une idée précise de cette importance. De surcroît, certaines références bibliques manquent dans la traduction (fr. $14: G n 2,16$ et 3, 3 ; fr. $18: G n 1$, 2 ; fr. 19 : Dt 4, 19 ; fr. 26 : Gn 1, 3.6.9 ; fr. $35: S g 7,21$; fr. $74: G n 9,3$; fr. 97 : $M t 11,19$ et $L c 7,34$ ). Il faudrait aussi corriger quelques références (le fr. 65 ne cite pas $O s$ 13, 4 mais $I s 43,11$; le fr. 93 qui fait allusion au jeûne de Moise ne renvoie pas à $E x 24,18$ mais à $E x 34,28$ ). L'ajout de guillemets dans le fr. 73 rapportant l'épisode de Phinees aurait permis de montrer que Julien ne se contente pas de raconter l'histoire mais reprend textuellement des expressions bibliques, comme il le signale lui-même en disant $\varphi \eta \sigma \mathrm{l}$. À propos du texte biblique cité par Julien, une remarque de l'introduction est assez étonnante (p. 34) : «Les citations qui parsèment son ouvrage sont fidèles et conformes, à quelques détails près, au texte que nous lisons aujourd'hui dans la Bible grecque ». Mais de quelle «Bible grecque » parle-t-on ? Outre qu'il peut y avoir des citations de mémoire, la comparaison des leçons de Julien doit être menée avec toutes les variantes de la tradition manuscrite de la Septante. L'annotation n'est pas toujours

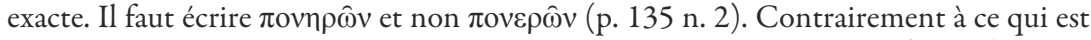
dit, le « symbole des Apôtres » n’a pas été défini par le concile de Nicée (p. 147).

En dépit de ces remarques, il faut saluer l'intérêt de cette publication qui fournit dans un format accessible, y compris financièrement, une traduction française rigoureuse et très lisible d'un ouvrage majeur, comme en témoigne sa postérité tant dans l'Antiquité tardive que jusqu'à l'époque moderne.

Marie-Odile Boulnois École Pratique des Hautes Études, Université PSL, LEM

Frédéric FAUQuIER, Le Parménide au miroir des platonismes. Logique-Ontologie-Théologie Paris, Les Belles Lettres, 2018 (Collection d'études anciennes. Série grecque, 157), 546 p., ISBN 978-2-251-44827-5.

Interroger la diversité des lectures antiques du Parménide pour dégager l'unité et la singularité du néoplatonisme, telle est l'ambition, pleinement tenue, du beau livre 
de Frédéric Fauquier. Celui-ci se donne comme une passionnante enquête métaexégétique, qui ne se contente pas de retracer l'histoire des interprétations mais en dégage les stratégies et la visée. « Dès son origine, écrit F. Fauquier, le néoplatonisme est tradition, puisqu'il projette son origine dans l'œuvre de Platon » (p. 317). Le livre s'emploie à montrer comment opère cette projection, quels choix, quelles torsions, quels coups de force elle engage, mais aussi à élucider, à partir d'eux, le geste herméneutique commun qui fonde le néoplatonisme en tant que tel. C'est en effet son rapport spécifique au Parménide qui constitue l'école néoplatonicienne en « famille spirituelle » - pour

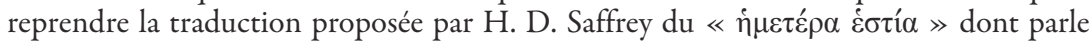
Proclus lorsqu'il évoque ce dialogue comme celui qui, « chez les amoureux de Platon, allume la parfaite et totale lumière de la théologie » (Théol. Plat. I, 7, p. 31.7-32.9). «Dialogue-miroir », le Parménide est ainsi le « lieu où s'invente le néoplatonisme comme phénomène unitaire » (p. 25).

Car c'est là, Frédéric Fauquier le rappelle d'emblée, ce qui fait la différence entre commentaire antique et commentaire moderne : le commentaire antique est un authentique exercice philosophique, qui cherche non seulement la vérité du texte mais « la vérité portée par le texte » (p. 18). Le choix du texte-source détermine ainsi l'orientation et l'unité problématique d'un courant ou d'une école de pensée : le médioplatonisme s'origine dans la lecture du Timée, comme le néoplatonisme dans celle du Parménide. À partir de là, il s'agit de considérer, au moins autant que le Parménide « au miroir » des platonismes, les platonismes « au miroir » du Parménide : de montrer comment la relation d'élection à ce texte détermine bien des moments philosophiques distincts - des platonismes, donc - et faits de ruptures par rapport à la tradition, indissociablement herméneutique et philosophique, qui les a précédés. Identifier ces ruptures, c'est isoler les conditions d'émergence du néoplatonisme et réaffirmer l'originalité de cette « famille spirituelle ».

Frédéric Fauquier dégage ainsi les principes qui, par-delà leurs divergences internes, vont constituer le cadre herméneutique commun aux différents auteurs néoplatoniciens: la polarisation sur la seconde partie du dialogue ; le découpage, en son sein, d'unités argumentatives, ou encore d' « hypothèses »; la corrélation stricte établie entre cette structure formelle et la structure même du réel.

Des principes ainsi dégagés, F. Fauquier déploie la double puissance de rupture et de fondation. La première partie de l'ouvrage est consacrée à la critique, par Proclus, des interprétations logiques du Parménide - particulièrement de celle en cours dans le médioplatonisme, avec Alcinoos, qui lit le dialogue comme un « réservoir de catégories » en même temps que comme une anticipation de la syllogistique. À une telle lecture, qui repose sur une « interaction herméneutique » entre d'une part le Parménide, d'autre part les Topiques et les Catégories, Proclus en oppose une autre qui, elle, croise le Parménide avec les livres VI et VII de la République, ainsi qu'avec le Sophiste et le Phèdre, de façon à « suturer » la méthode par hypothèses aux quatre opérations de la dialectique et à la renvoyer à la visée de l'anhypothétique. Cette stratégie proclusienne est soutenue par un « noyau dur ou [...] un impératif herméneutique irréductible »: l'hypothèse «si l'un est » décrit une réalité (p. 127). F. Fauquier souligne les «torsions »- notamment, la multiplication des hypothèses - que cette lecture impose au texte-source, et qui à leur tour contraignent Proclus à mobiliser la théorie des hénades comme intermédiaires entre l'un et le multiple.

La deuxième partie de l'ouvrage s'attache au dépassement des interprétations ontologiques du Parménide, notamment de celle - inscrite, là encore, dans le cadre d'un médioplatonisme aristotélisé - d'Origène le platonicien, qui fait de l'intellect le premier 
principe et refuse la position de l'un au-delà de l'être. Contre cette lecture, il s'agit de montrer que la première hypothèse a un objet réel, ou encore, et comme l'écrit Proclus,

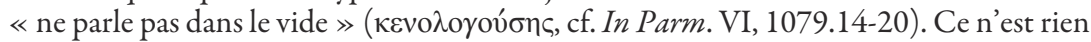
de moins que le geste fondateur du néoplatonisme qui se joue là, soit l'extension de la réalité au-delà de l'être. F. Fauquier en analyse les différentes déclinaisons chez Proclus, encore, mais aussi chez Plotin, Porphyre, Jamblique et Syrianus, tout en montrant comment, une nouvelle fois, cette décision herméneutique implique de faire violence au texte-source et particulièrement de redécouper et réinterpréter la conclusion de la première hypothèse (Parm. 142a6-8) qui paraît l'invalider.

Une troisième et dernière partie, très ample, interroge enfin ce qui constitue le cœur même de l'interprétation néoplatonicienne du Parménide : soit indissociablement la lecture de la première hypothèse comme lieu « où se joue l'exposition d'une transcendance radicale » et la lecture théologique de la deuxième hypothèse. F. Fauquier dégage les trois réquisits d'une telle lecture, ainsi que les différentes modalités de leur mise en œuvre chez Syrianus, Proclus, Jamblique et Damascius : identifier l'un avec le principe de toute chose ; penser que la théologie peut s'exposer sous une forme dialectique et que les négations peuvent soutenir le discours théologique ; enfin, considérer le texte platonicien comme porteur d'une vérité (p. 196). Une vérité d'ailleurs non exclusive puisque - et c'est un point que F. Fauquier interroge également - le néoplatonisme postérieur à Plotin va accorder aux Oracles chaldä̈ques une place elle aussi centrale, s'imposant dès lors de nouvelles « contorsions herméneutiques » de façon à articuler ce texte de structure médioplatonicienne à une hénologie radicale.

Selon la méthode déjà mise en oeuvre, et qui oriente son projet, F. Fauquier s'attache à la fois à analyser en détail la mise en œuvre de ces présupposés herméneutiques, et à souligner la rupture novatrice qu'ils impliquent. De fait, demander si l'on en trouve trace avant Plotin - ainsi, chez Modératus, Numénius, ou les Gnostiques - c'est là encore interroger à nouveaux frais l'originalité du néoplatonisme, la pertinence de sa distinction d'avec le médioplatonisme, et, de ce fait, la pluralité effective des platonismes. À cette question, la réponse de F. Fauquier est claire : si l'exégèse du Parménide est moins centrale chez Plotin que chez ses successeurs, elle est bien radicalement novatrice, et dégage les acquis fondamentaux qui, légués et amplifiés, vont constituer le néoplatonisme en nouvelle « famille spirituelle » et inventer une « configuration métaphysique inédite ». Pour autant, cette lecture théologique est, écrit F. Fauquier, « la moins évidente : elle fait presque violence au texte platonicien dans lequel elle projette une pensée de la transcendance radicale » (p. 428). L'un des grands mérites de cette belle enquête, qui conjugue l'amplitude de champ à la précision de l'analyse (précision dont témoignent encore les études de détail rassemblées en 16 annexes), est d'interroger cette violence qui, parce qu'elle voit en lui la source d'une vérité à extraire et à déployer, fait d'un texte l'origine d'une tradition.

Gwenaëlle Aubry Centre Jean Pépin UMR 8230

Fulvia DE Luise (éd.), Cittadinanza. Inclusi ed esclusi tra gli antichi e i moderni, Trento, Università degli Studi di Trento, 2018 (Studi e Ricerche, 17), 297 p., ISBN 978-88-8443-798-3.

L'homme antique reste souvent identifié à la figure de l'homo politicus, et plus précisément à celle du « citoyen ». Mais que désigne ce terme, transmis jusqu’à nous comme si sa signification n'avait pas changé ? Les neuf textes réunis dans ce volume, 